\title{
Study on the relationship between Cardio- cerebrovascular events and the level of Atherosclerosis evaluated by Heart-brain Integrated CTA
}

Huan Yu ( $\sim$ 29222788@qq.com )

Capital Medical University

Yue Zhang

Chengde Medical University

Dong Zhu

Chengde Medical University Affiliated Hospital

Kuai Duan

Capital Medical University

Yi Hu

Capital Medical University

Chunhua Song

Capital Medical University

\section{Research}

Keywords: CTA scan, cardio-cerebrovascular, Cardio-cerebrovascular disease, Vascular stenosis

Posted Date: January 21st, 2020

DOI: https://doi.org/10.21203/rs.2.21386/v1

License: (c) (1) This work is licensed under a Creative Commons Attribution 4.0 International License.

Read Full License 


\section{Abstract}

Background: To establish a one-stop cardio-cerebral vascular scanning technique based on doublesource helical CT by analyzing the relationship between the nature of coronary artery, extracranial and extracranial vascular plaque, the degree of lumen stenosis and the occurrence and development of cardio-cerebrovascular disease (CCVD).

Methods: the plaque properties, vascular stenosis degree, and plaque quantitative analysis results of coronary artery, extracranial and intracranial segments of coronary artery, extracranial and intracranial carotid artery in 39 patients who underwent combined heart-brain CTA scanning were analyzed. The correlation between the load degree of cardio-cerebrovascular plaque and cardio-cerebrovascular events was analyzed comprehensively.

Results: there were significant differences in age, high-density lipoprotein, diabetes, hypertension, smoking, plaque nature, plaque location, plaque composition and NIHSS score between the two groups.

Conclusion: the combination of heart and brain CTA can help to establish the best clinical predictive model to prevent and stop the occurrence and development of coronary heart disease and stroke in time.

\section{Background}

In recent years, stroke has become the number one killer threatening our national health. According to the results of a series of studies on the global burden of disease in 2018, despite the general increase in global life expectancy, the combined disease burden is getting heavier and heavier, and stroke has become the second leading cause of death in the world's population ${ }^{1,2}$. From a worldwide point of view, China is a high-risk area of stroke in the world. From a domestic point of view, stroke has replaced heart disease as the main cause of death and disability of adults in China. We believe that lumen stenosis, plaque shedding, and intra-plaque hemorrhage caused by carotid atherosclerosis are the main causes of stroke ${ }^{3}$. Some experiments have proved that there is a close relationship between carotid atherosclerosis and coronary artery atherosclerosis ${ }^{4}$. Nowadays, the concept of prevention has gradually taken root in the hearts of the people, and the main battlefield of cardio-cerebrovascular diseases has been gradually transformed into primary prevention ${ }^{5}$. The purpose of this study is to establish a one-stop cardio-cerebral macrovascular scanning process based on dual-source spiral CT and to establish a standard for evaluating plaque load. To establish a risk model of cardiovascular and cerebrovascular recurrence of extracranial and extracranial large artery stenosis based on whole-body plaque load measurement. Timely prevent and stop the occurrence and development of coronary heart disease and stroke.

\section{Results}

\subsection{General information}


Among the 39 patients in this study, there were 22 males (0.56\%) and 17 females (44\%). The age was $42 \mathrm{mi} 83$ years old, with an average of $65.6 \pm 12.5$ years old. According to the occurrence of cardiocerebrovascular events, patients were divided into two groups: patients with cardio-cerebrovascular events and those without cardio-cerebrovascular events.

\subsection{CTA check result}

There were 149stable plaques and 124unstable plaques in 39 patients by CTA. The CT value of calcified plaque was $(523 \pm 242) \mathrm{HU}$, and that of the mixed plaque was $(97 \pm 39) \mathrm{HU}$, The CT value of non-calcified plaque was $(25 \pm 32) \mathrm{HU}$. CTA showed mild stenosis (145cases), moderate stenosis (27cases), severe stenosis (12cases), and occlusion (3cases).

Table 1 CTA shows plaque properties and stenosis statistics

Plaque property Normal Degree of stenosis Total

\begin{tabular}{|c|c|c|c|c|c|c|}
\hline & & & & & & \\
\hline & & Mild & Moderate & Severe & Block & \\
\hline Stable plaque & 52 & 83 & 13 & 1 & 0 & 149 \\
\hline Unstable plaque & 34 & 62 & 14 & 11 & 3 & 124 \\
\hline Total & 86 & 145 & 27 & 12 & 3 & 273 \\
\hline
\end{tabular}

\subsection{Cox regression analysis}

Univariate analysis showed that there were significant differences in age, high-density lipoprotein, diabetes, hypertension, smoking, plaque nature, plaque location, plaque composition, and NIHSS score between cardio-cerebrovascular event group and non-cardio-cerebrovascular event group. (Table 2)

Table 2 Cox regression analysis of cardio-cerebrovascular events 
Univariate analysis

Hazard ratio p value
$(95 \% \mathrm{Cl})$

Multivariable analysis

Model $1^{\mathrm{a}} \quad$ Model $2^{\mathrm{a}}$

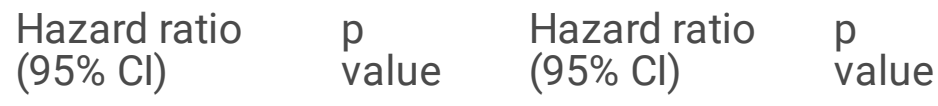

\section{Demographics}

\begin{tabular}{|c|c|c|c|c|c|c|}
\hline Age & $\begin{array}{l}0.86(0.81- \\
0.95)\end{array}$ & $<0.001$ & $\begin{array}{l}0.85(0.82- \\
0.95)\end{array}$ & 0.006 & $\begin{array}{l}0.90 \rrbracket 0.83- \\
0.98 \rrbracket\end{array}$ & 0.012 \\
\hline Sex囚men & $\begin{array}{l}1.15 \rrbracket 0.83- \\
1.53 \rrbracket\end{array}$ & 0.443 & $\begin{array}{l}1.33 \otimes 0.98- \\
1.81 \rrbracket\end{array}$ & 0.068 & $\begin{array}{l}1.29 ه 0.95- \\
1.76 \rrbracket\end{array}$ & 0.100 \\
\hline
\end{tabular}

\section{Laboratory}

findings

\begin{tabular}{|c|c|c|c|c|c|c|}
\hline $\begin{array}{l}\text { Hemoglobin, } \\
\text { g/dL }\end{array}$ & $\begin{array}{l}1.0481 .02- \\
1.06 \rrbracket\end{array}$ & $<0.001$ & $\begin{array}{l}1.02(1.01- \\
1.03)\end{array}$ & 0.006 & $\begin{array}{l}1.02(1.01- \\
1.03)\end{array}$ & 0.007 \\
\hline Total cholesterol & $\begin{array}{l}0.82(0.72- \\
0.97)\end{array}$ & 0.002 & $\begin{array}{l}0.93(0.82- \\
1.05)\end{array}$ & 0.368 & $\begin{array}{l}0.93(0.81- \\
1.07)\end{array}$ & 0.324 \\
\hline HDL cholesterol & $\begin{array}{l}0.74(0.45- \\
1.20)\end{array}$ & 0.205 & & & & \\
\hline LDL cholesterol & $\begin{array}{l}0.93(0.78- \\
1.06)\end{array}$ & 0.186 & & & & \\
\hline
\end{tabular}

\section{Medication at}

discharge

$\begin{array}{lll}\text { Statin } & 0.54(0.21- & 0.235 \\ & 1.42) & \end{array}$

$\begin{array}{lll}\text { Antihypertensive } & 0.73(0.44- & 0.164 \\ & 1.16)\end{array}$

$\begin{array}{lll}\text { Anticoagulant } & 1.13(0.83- & 0.456 \\ & 1.49) & \end{array}$

\section{Risk factors}

\begin{tabular}{|c|c|c|c|c|c|c|}
\hline Hypertension & $\begin{array}{l}0.73(0.43- \\
1.17)\end{array}$ & 0.164 & & & & \\
\hline Diabetes mellitus & $\begin{array}{l}1.10(0.81- \\
1.54)\end{array}$ & 0.451 & & & & \\
\hline Atherosclerosis & $\begin{array}{l}1.05(0.97- \\
1.09)\end{array}$ & 0.145 & & & & \\
\hline INIHSS score & $\begin{array}{l}2.33(1.75- \\
3.19)\end{array}$ & $<0.001$ & $\begin{array}{l}1.92(1.47- \\
2.63)\end{array}$ & $<0.001$ & $\begin{array}{l}1.80(1.33- \\
2.49)\end{array}$ & $<0.001$ \\
\hline Current smoker & $\begin{array}{l}1.02(1.00- \\
1.09)\end{array}$ & $<0.001$ & $\begin{array}{l}1.03(1.00- \\
1.06)\end{array}$ & 0.002 & $\begin{array}{l}1.06(1.02- \\
1.09)\end{array}$ & 0.002 \\
\hline
\end{tabular}




\section{Extent of stenosis}

\begin{tabular}{|c|c|c|c|c|c|c|}
\hline No stenosis & 1 & & 1 & & & \\
\hline Mild stenosis & $\begin{array}{l}1.36(0.98- \\
2.05)\end{array}$ & 0.084 & $\begin{array}{l}1.24(0.84- \\
1.88)\end{array}$ & 0.201 & & \\
\hline $\begin{array}{l}\text { Moderate } \\
\text { stenosis }\end{array}$ & $\begin{array}{l}1.43(0.94- \\
2.29)\end{array}$ & 0.073 & $\begin{array}{l}1.35(0.96- \\
2.19)\end{array}$ & 0.142 & & \\
\hline Severe stenosis & $\begin{array}{l}2.56(1.62- \\
4.00)\end{array}$ & $<0.001$ & $\begin{array}{l}2.25(1.35- \\
3.52)\end{array}$ & 0.001 & & \\
\hline Block & $\begin{array}{l}4.10(2.60- \\
6.47)\end{array}$ & $<0.001$ & $\begin{array}{l}2.92(1.80- \\
4.60)\end{array}$ & $<0.001$ & & \\
\hline \multicolumn{7}{|l|}{ Plaque property } \\
\hline Stable plaque & $\begin{array}{l}1.35(0.93- \\
1.95)\end{array}$ & 0.087 & & & $\begin{array}{l}1.25(0.87- \\
1.83)\end{array}$ & 0.215 \\
\hline Unstable plaque & $\begin{array}{l}2.21(1.59- \\
3.10)\end{array}$ & $<0.001$ & & & $\begin{array}{l}2.93(1.82- \\
4.60)\end{array}$ & $<0.001$ \\
\hline
\end{tabular}

Model $1^{\mathrm{a}}$ included the presence of CCVD as entering variables, whereas Model $2^{\mathrm{a}}$ used the extent of CCVD.

\section{4 quantitative analysis of plaques}

All patients' images were measured and quantitatively analyzed by the modified APQ (AUTOPLAQ) technique (figure 1). The results of plaque quantitative analysis (Table 3 ) were compared in patients with the symptomatic cardio-cerebrovascular disease ((CCVD)). Based on the evaluation of systemic atherosclerosis, the risk stratification index of cardiovascular events in symptomatic CCVD patients was established.

Fig. 1 Measurement using improved APQ technique Lesion quantification: Red and orange for NCP, orange for Low-density NCP $<30 \mathrm{HU}$, Yellow for CP.

\section{Table 3 quantitative analysis of plaques}




\begin{tabular}{|c|c|c|c|}
\hline Patch feature & $\begin{array}{l}\text { No cardio-cerebrovascular } \\
\text { events occurred }\end{array}$ & $\begin{array}{l}\text { The occurrence of cardio- } \\
\text { cerebrovascular events }\end{array}$ & $\begin{array}{l}\mathrm{P}- \\
\text { value }\end{array}$ \\
\hline NCP Volume & $41.23 \pm 25.34$ & $262.5 \pm 100.43$ & 0.038 \\
\hline $\mathrm{CP}$ Volume & $8.54 \pm 3.44$ & $11.4 \pm 4.75$ & 0.288 \\
\hline LD NCP Volume & $35.65 \pm 17.48$ & $66.3 \pm 24.53$ & 0.011 \\
\hline $\begin{array}{l}\text { Total Plaque } \\
\text { Volume }\end{array}$ & $66.3 \pm 12.76$ & $273.9 \pm 43.87$ & $<0.001$ \\
\hline NCP Burden $\otimes \% \bigotimes$ & $23.6 \pm 14.32$ & $47.6 \pm 12.45$ & 0.001 \\
\hline $\begin{array}{l}\text { LD NCP Burden } \\
\varangle \% \bigotimes\end{array}$ & $7.32 \pm 3.45$ & $12.0 \pm 6.23$ & 0.021 \\
\hline CP Burden $\nabla \% \rrbracket$ & $1.0 \pm 1.51$ & $2.1 \pm 1.21$ & 0.018 \\
\hline $\begin{array}{l}\text { Total Plaque } \\
\text { Burden } \otimes \% \square\end{array}$ & $46.92 \pm 29.50$ & $61.7 \pm 15.6$ & 0.001 \\
\hline Remodeling Index & $1.09 \pm 0.32$ & $3.33 \pm 0.55$ & 0.014 \\
\hline $\begin{array}{l}\text { Diamter Stenosis } \\
\bigotimes \% \bigotimes\end{array}$ & $20.51 \pm 18.32$ & $33.2 \pm 24.98$ & 0.021 \\
\hline Area stenosis $₫ \% \bigotimes$ & $23.58 \pm 14.65$ & $57.2 \pm 38.37$ & 0.002 \\
\hline Plaque Length & $2.25 \pm 1.83$ & $4.56 \pm 4.19$ & 0.004 \\
\hline $\begin{array}{l}\text { Contrast density } \\
\text { drop } ₫ \otimes \square\end{array}$ & $25.65 \pm 6.31$ & $35.1 \pm 5.87$ & 0.034 \\
\hline
\end{tabular}

\section{Discuss}

The nature of atherosclerotic plaque and lumen stenosis are significantly related to the occurrence of cardio-cerebrovascular events ${ }^{6}$. Therefore, by detecting and judging the degree of atherosclerosis, especially the stability of plaque, and combining with clinical NIHISS score and cardio-cerebrovascular risk factors, it is of great significance for clinical guidelines to prevent the occurrence of cardiocerebrovascular events.

In the process of clinical diagnosis, a one-stop CTA can well reflect the degree of atherosclerosis ${ }^{7}$. And one-stop CTA has the advantages of short scanning time, low radiation dose, and good safety, which makes it an inspection method with broad application prospects ${ }^{8}$. The improved APQ technique can more accurately analyze the non-calcified components in the plaque so that the plaque parameters can be obtained more accurately, which is helpful to comprehensively evaluate the plaque load of the coronary artery and head-carotid artery. The commonly used clinical calcification score CACS refers to Agatston 
score $^{9}$, its disadvantage is due to the partial volume effect, the small calcification plaque score is easy to change during reexamination, the improved $A P Q$ technique avoids this change. Keelan ${ }^{10}$ et al. pointed out that calcification score can be used as an independent predictor of future coronary events, and is superior to the degree of stenosis and traditional risk factors. There is also a strong correlation between coronary arteries and head and neck vessels ${ }^{11}$. Cardio-cerebral combined CT angiography is a one-stop cardio-cerebrovascular combined scanning on the basis of CTA, which greatly improves the image resolution, greatly shortens the scanning time and reduces the radiation dose; it can be used to scan the cardio-cerebral vessels of patients to meet the clinical needs.

Some experiments have proved that carotid artery disease has a high predictive value for coronary artery disease ${ }^{12}$. Considering the characteristics of ultrasonic detection of carotid artery, such as non-invasive, convenient, repeatable and relatively cheap, when planning to use heart-brain integrated CTA to evaluate coronary artery and carotid artery disease, it is reasonable to exclude traditional risk factors for ultrasonic detection of carotid artery in advance, and it is not recommended or recommended to apply heart-brain integrated CTA to the physical examination of people without indications. In patients with multiple hard plaques or mixed plaques in the carotid artery, the examination of integrated heart-brain CTA may be more targeted ${ }^{13}$.

Our study found that the more risk factors of cardiovascular and cerebrovascular diseases such as male, older, smoking, hypertension, diabetes, hyperlipidemia, the greater the probability of cardiovascular and cerebrovascular events, the more likely it is to induce cardio-cerebrovascular events.

There are still many shortcomings in this study, such as limited sample size, the low exposure level of cardio-cerebrovascular diseases, lack of long-term follow-up, and follow-up comparison of imaging and clinical data.

In summary, this study used integrated heart-brain CTA scan to establish a risk prediction model for patients with symptomatic intracranial and extracranial stenosis based on whole-body plaque load score, so as to realize individualized treatment of intracranial arterial stenosis, optimize the allocation of medical resources, optimize the process of stroke prevention and treatment, improve medical efficiency and reduce stroke incidence and recurrence rate, which is of great significance to the secondary prevention of stroke.

\section{Conclusion}

We found that NCPVolume,TotalPlaqueBurden (\%), Remodelinglndex) is the risk factor of cardiocerebrovascular events, among which TotalPlaqueBurden has the greatest risk. The combination of the three can be widely used in clinical practice to predict cardio-cerebrovascular events.

\section{Methods}




\subsection{Object}

A total of 39 patients with suspected coronary heart disease or stroke who were admitted to our hospital in 2018.06 /2019.05 were examined by combined heart-brain CTA, and all patients were treated with standardized and optimized drug therapy. All patients were followed up at 3 and 6 months after onset, and the medication, vital signs, and cardiovascular and cerebrovascular events (ischemic stroke, coronary syndrome, vascular death) were recorded. All patients completed relevant laboratory tests within 24 hours of admission, such as blood glucose, HbA1C, D-dimer, four items of blood coagulation, LDL-C, HDL-C, TG, $\mathrm{TCH}$, biochemical markers (CRP, hs-CRP, MMP2, MMP9, oxLDL-C, etc.). The inclusion criteria were: (1) age: $18 \mathrm{Mel} 80$ years old; (2) auxiliary examination (including carotid ultrasound TCD, head, and neck CTA or MRA) indicating intracranial artery stenosis; (3) patients with clinical symptoms suspected of coronary heart disease; (4) $\geq$ two atherosclerotic risk factors (5) informed consent of patients and their families. The exclusion criteria were as follows: (1) patients with irregular heart rate could not complete combined heart and brain CTA examination (2) patients with PFO, atrial fibrillation, connective tissue disease, tumor and other non-atherosclerotic stroke, (3) head CT or MRI indicated hemorrhage, watershed infarction, or other diseases (vascular malformation, tumor, brain abscess, etc.); (4) patients with previous history of gastrointestinal ulcer who could not tolerate dual anti-platelet aggregation therapy with aspirin and clopidogrel, (5) patients with decreased liver and renal function (ALT, AST $\geq 3$ times normal high limit or creatinine $\mathrm{F}>1.5 \mathrm{mg} / \mathrm{dl}$ ) or increased CK ( $\geq 10$ times normal high limit); (6) allergic to aspirin, clopidogrel and statins, or could not be tolerated.

\subsection{Inspection method}

Siemens dual-source CT was used for large pitch scanning, the scanning range was from the other side to the top of the head, and the direction of the foot was scanned. FlashSpira mode is adopted. Pitch: 3.2, tube voltage 70 mur90kV, tube current $330 \mathrm{Mur} 450 \mathrm{mAs}$, rotating speed $0.25 \mathrm{~s} / \mathrm{circle}$, collimation width: $0.6 \mathrm{~mm} * 96$. Contrast agent iohexol $50 \mathrm{ml}$ was injected intravenously with $5 \mathrm{ml} / \mathrm{s}$, followed by saline $50 \mathrm{ml}$ at the same rate. The aortic root was selected to set the threshold for ROI, to $100 \mathrm{HU}$, and the contrast tracer technique (Bolus-tracking method) was used to detect the density change of RIO. When the CT value of the RIO exceeded the threshold, the scan was triggered automatically with a delay of 8 seconds. The heart scan ECG triggers $30 \%$ or $65 \%$ of the cardiac cycle.

\subsection{Image reconstruction and data post-processing}

The collected data were processed by ADW4.6 workstation for various post-processing images, including volume rendering (VR), maximum density projection (MIP), curved surface reconstruction (CPR) reconstruction layer thickness of $0.75 \mathrm{~mm}$ and spacing of $0.4 \mathrm{~mm}$. The best quality CTA images were analyzed for analysis and diagnosis. At the same time, the plaques of blood vessels were measured and quantitatively analyzed by the modified APQ (AUTOPLAQ) technique in all patients' images. The branch of the coronary artery is divided into three parts: the left trunk and left anterior descending branch, left circumflex branch, and the right coronary artery. The head and carotid artery is divided into four parts: the extracranial segment of the right head-carotid artery, the extracranial segment of the left head-carotid 
artery, the intracranial segment of the right head-carotid artery, and the extracranial segment of the left head-carotid artery, including the aortic arch, the subclavian artery, the common carotid artery, the extracranial segment of the internal carotid artery and the extracranial segment of the vertebral artery. The intracranial segment of the cephalic carotid artery includes basilar artery, bilateral internal carotid artery, vertebral artery and anterior, middle and posterior cerebral arteries. For vessels with stenosis, the vessel diameter is measured by APQ vascular analysis software, and then according to the degree of stenosis, it can be divided into severe stenosis group (stenosis rate $>75 \%$ ), moderate stenosis group (stenosis rate $50 \%-74 \%$ ), mild stenosis group (stenosis rate $<50 \%$ ). Vascular stenosis $\geq 50 \%$ was defined as meaningful stenosis, and the degree of vascular stenosis was calculated. The degree of stenosis $=(1$ the lumen area of the lesion site / the lumen area of the control site) $\times 100 \%$. It is found that the risk of plaque composition and its stability for atherosclerosis is much higher than that of arterial lumen stenosis ${ }^{14}$, so we further analyze the composition of plaque. Using the improved APQ non-calcified plaque detection technique, the plaque was divided into calcified plaque, non-calcified plaque and mixed plaque. We define non-calcified plaques and mixed plaques as unstable plaques. The indexes of noncalcified plaques were evaluated comprehensively.

\subsection{Statistical analysis}

SPSS statistical software was used to integrate and process the data. The measurement data were expressed as " $\mathrm{x} \pm \mathrm{s}$," the t-test of independent samples was adopted, and the counting data were analyzed by $x^{2}$,Cox regression method to analyze the correlation between various factors and cardiocerebrovascular diseases.

\section{Declarations}

\section{Abbreviation}

CCVD:cardio-cerebrovascular disease;NIHSS: National Institute of Health stroke scale.

\section{Acknowledgements}

The authors wish to thank Dr. Duan. Director of radiology department, Liangxiang teaching hospital, Capital Medical University, for allowing experiments to be conducted with the patients.

\section{Authors' contributions}

Huan Yu designed the experimental procedures and submitted the plan of the study to the ethical committee, then supervised the research carried out. Yue Zhang,Dong Zhu designed and conducted analyses based upon data methods. Kuai Duan1,Yi Hu,Chunhua Song contributed to "Background" and "Discussion" sections. All authors read and approved the final manuscript.

\section{Funding}


The project was supported by the Hospital level project of Beijing Liangxiang Hospital number2018-02.

\section{Availability of data and materials}

The datasets used and/or analyzed during the current study are available from the corresponding author on reasonable request.

\section{Ethics approval and consent to participate}

The research plan has been submitted to the ethics committee of Liangxiang Hospital, Fangshan District, Beijing for approval. The Committee issued a positive decision No. 2018-02 on August 31, 2018. The ethics committee of medical college is part of a large medical university in Beijing. The written informed consent for participation was obtained from the caregivers and relatives of all participants.

\section{Consent for publication}

Not applicable.

\section{Competing interests}

The authors declare that they have no competing interests

\section{References}

1. Hertz JT, Sakita FM, Limkakeng AT, Mmbaga BT, Appiah LT, Bartlett JA, et al. The burden of acute coronary syndrome, heart failure, and stroke among emergency department admissions in tanzania: A retrospective observational study. African journal of emergency medicine : Revue africaine de la medecine d'urgence. 2019;9:180-184

2. Hu T, Gu Y, Xu Y, Yu J, Wu F, Chen R. Incidence of stroke and mortality in chinese patients with sleepbreathing disorders: A clinical population-based (cpb) study. Medical science monitor: international medical journal of experimental and clinical research. 2019;25:10129-10135

3. Howard BM, Hu R, Barrow JW, Barrow DL. Comprehensive review of imaging of intracranial aneurysms and angiographically negative subarachnoid hemorrhage. Neurosurgical focus. 2019;47:E20

4. Mammo DF, Cheng $\mathrm{Cl}$, Ragina NP, Alani F. Factors affecting cardiovascular and cerebrovascular complications of carotid artery stenting in northern michigan: A retrospective study. Cardiovascular revascularization medicine : including molecular interventions. 2017;18:S18-s21

5. Millon A, Mathevet JL, Boussel L, Faries PL, Fayad ZA, Douek PC, et al. High-resolution magnetic resonance imaging of carotid atherosclerosis identifies vulnerable carotid plaques. Journal of vascular surgery. 2013;57:1046-1051.e1042 
6. St Pierre S, Siegelman J, Obuchowski NA, Ma X, Paik D, Buckler AJ. Measurement accuracy of atherosclerotic plaque structure on ct using phantoms to establish ground truth. Academic radiology. 2017;24:1203-1215

7. Ognard J, Dissaux B, Haioun K, Nonent M, Gentric JC, Ben Salem D. A "one-stop-shop" 4d cta protocol using 320-row ct for advanced imaging in acute ischemic stroke: A technical note. European radiology. 2019;29:4930-4936

8. Ni QQ, Tang CX, Zhao YE, Zhou CS, Chen GZ, Lu GM, et al. Single phase dual-energy ct angiography: One-stop-shop tool for evaluating aneurysmal subarachnoid hemorrhage. Scientific reports. 2016;6:26704

9. Sandstedt M, Henriksson L, Janzon M, Nyberg G, Engvall J, De Geer J, et al. Evaluation of an aibased, automatic coronary artery calcium scoring software. European radiology. 2019

10. Keelan PC, Bunch TJ, White RD, Packer DL, Holmes DR, Jr. Early direct coronary angioplasty in survivors of out-of-hospital cardiac arrest. The American journal of cardiology. 2003;91:1461-1463, a1466

11. Oron Y, Shemesh S, Shushan S, Cinamon U, Goldfarb A, Dabby R, et al. Cardiovascular risk factors among patients with vestibular neuritis. The Annals of otology, rhinology, and laryngology. 2017; 126:597-601

12. Tani $H$, Sawano M, Numasawa Y, Kobayashi Y, Suzuki M, Noma S, et al. In-hospital outcome in patients presenting with acute coronary syndrome with left main coronary artery disease: A report from japanese prospective multicenter percutaneous coronary intervention registry. Journal of cardiology. 2019

13. Yuksel B, Koc P, Ozaydin Goksu E, Karacay E, Kurtulus F, Cekin Y, et al. Is multiple sclerosis a risk factor for atherosclerosis? Journal of neuroradiology = Journal de neuroradiologie. 2019

14. Saxena A, Ng EYK, Lim ST. Imaging modalities to diagnose carotid artery stenosis: Progress and prospect. Biomedical engineering online. 2019;18:66

\section{Figures}




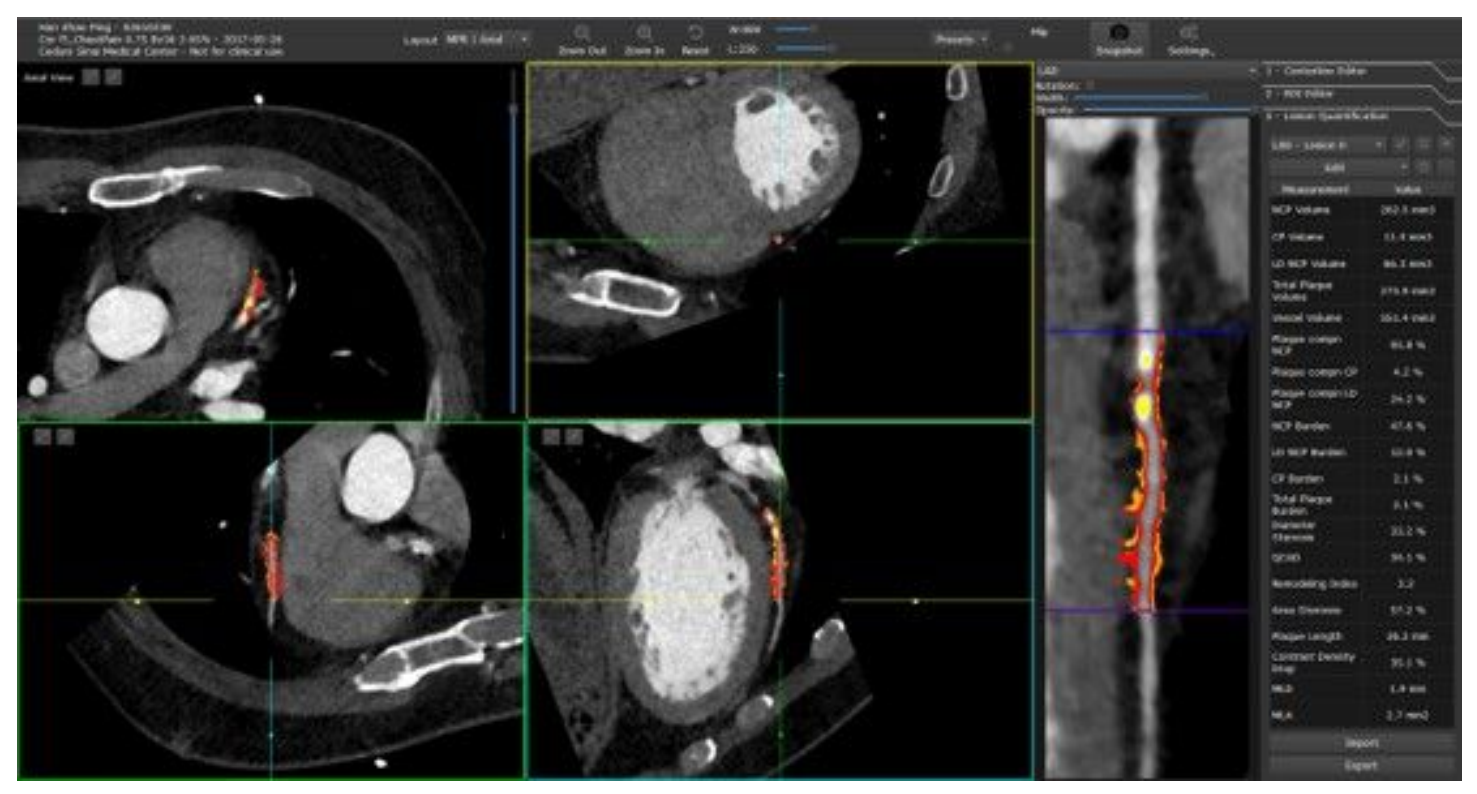

\section{Figure 1}

Measurement using improved $\mathrm{APQ}$ technique Lesion quantification: Red and orange for NCP, orange for Low-density NCP $<30 \mathrm{HU}$, Yellow for CP. 